\title{
IMPROVEMENT OF SYMPTOMS OF CHRONIC PROSTATITIS LIKE PERINEAL HEAVINESS AND FREQUENCY AFTER TEN COMPLETE SESSION OF PER RECTAL INFRA RED LASER THERAPY
}

\section{ABDUL MATIN ANAMUR RASHID CHOUDHURY ${ }^{1}$, MOHAMMED MIZANUR RAHMAN ${ }^{1}$, MD. WALIUL ISLAM ${ }^{1}$, TASMINA PARVEEN ${ }^{2}$, PARVEEN SULTANA ${ }^{3}$, MOHAMMAD ALAUDDIN ${ }^{4}$}

${ }^{1}$ Department of Urology, National Institute of Kidney Diseases and Urology, Dhaka, ${ }^{2}$ Department of Physiology, Uttara Adhunik Medical College, Uttara, Dhaka, ${ }^{3}$ Department of Forensic Medicine, Enam Medical College Hospital, ${ }^{4}$ Department of Anaesthesilogy, National Institute of Kidney Diseases and Urology, Dhaka.

\begin{abstract}
:
Objective : To observe the improvement of the symptoms of perineal heaviness and frequency after treatment with Magnetic Infra Red Laser therapy in patients of chronic pelvic pain syndrome /Chronic Prostatitis.

Methods : This study was an observational study done on 30 male patients with age range varied from 30 to 60 years. The study was conducted from 2009 to 2011 in a private medical center at Dhaka city. The MIL-therapy in use has a wave length of $904 \mathrm{~nm}$ and a frequency of $3000 \mathrm{~Hz}$. The Laser beam reaches the prostate with a special optical probe. In this study the patient is examined per rectally after doing digital rectal examination with use of gloves and gel. At the beginning we used a "Laser Super Sonic" machine with endorectal optical probe according to Strada. The treatment schedule was one treatment every two days (treatment's time of $5 / 10$ minutes, wave length $1000 \mathrm{KHz}$ ) for a total of 10 applications. After 10 applications patients were asked for follow up and data were collected and analysed.
\end{abstract}

Results : Out of 30 patients of chronic pelvic pain syndrome aged between 30-60 yrs were under treatment with MIL therapy. Among them only 22 patients were with complains of perineal heaviness, 20 patients were cured and 22 patients were with complains of frequency, 20 were cured and 02 were improved after laser treatment. Among 30 cases 23 patients found normal sized prostate and 07 found enlarged prostate on digital rectal examination and on USG findings, 21 were found normal sized, 09 found enlarged sized prostate and 07 patients had increased PVR.

Conclusion : Treatment with Magnetic infra red laser therapy (MIL-therapy) gives useful effect on chronic pelvic pain syndrome (CPPS). The following short article gives a very good summary of the benefits of laser therapy and totally cured of symptoms. CPPS, a painful type of Chronic Prostatitis frequently troubles man for years. Among many sign and symptoms of CPPS, like perineal heaviness and frequency were observed and found very good effect after laser therapy.

Key words : MIL Therapy ,Chronic pelvic pain syndrome (CPPS), Perineal heaviness, Frequency.

Bangladesh J. Urol. 2015; 18(2): 79-82

Correspondence : Abdul Matin Anamur Rashid Choudhury, Assistant Professor ,Resident Surgeon, Department of Urology,National Institute of Kidney Diseases and Urology. Dhaka, E-mail: dr-choudhury2001@yahoo.com 


\section{Introduction :}

Chronic prostatitis or chronic pelvic pain syndrome (CP/ CPPS) occurs in men and is characterized by persistent discomfort or pain in the pelvic area that lasts several months, often longer. The discomfort is usually at the base of the penis and around the anus and lower back. Sometimes it spreads into the testes. Some patients have perineal heaviness, and others may have pain or a frequency or urgency when they pass urine, as well as a poor urinary stream[1]. Laser means Light Amplification by Stimulated Emission of Radiation and was first theorized by Einstein. This was a tube laser with a metal chamber, which contained the element ruby[2,3]. MIL therapy is based on a combinative mutually reinforcing influence of impulse infrared laser irradiation, pulsating red light, pulsating wide-band infrared irradiation and static magnetic field on a biological object .Laser is a technical device, which emits in the form of a focused directive beam a coherent monochromatic polarized electromagnetic irradiation, i.e. the light in a very narrow spectral region. The cause of CP/CPPS is not known[3]. Prostatitis is a disease entity that is diagnosed by symptoms, microscopy of expressed prostatic secretions (EPS) and culture of EPS and segmented urine samples. It is a common medical condition $[4,5,6]$.

There are two main types of chronic prostatitis. 'Chronic bacterial prostatitis' is caused by persistent (chronic) infection with a germ (a bacterial infection). 'Chronic prostatitis/chronic pelvic pain syndrome (CPPS)' has a cause which is not fully understood[7]. Chronic prostatitis typically causes pain in the lower pelvic region of men. Urinary symptoms such as frequency of passing urine and pain on passing urine may also be present. Treatment can be difficult and may include antibiotics and other medicines. Symptoms may last a long time, although they may 'come and go' or vary in severity[8].

Chronic prostatitis/CPPS is a persistent (chronic) discomfort or pain that you feel in your lower pelvic region - mainly at the base of your penis and around your anus. It is usually diagnosed if you have had pain for at least three months within the previous six months. The cause of this type of chronic prostatitis is not fully understood. Many theories have been put forward as to the cause. These include:

- Infection of the prostate with a germ that has not yet been identified.

- Nerve problems affecting the prostate.

- An autoimmune problem of the prostate gland (antibodies that we normally produce to fight infection may be attacking the cells of the prostate gland for some reason).

Inflammation resulting from urine being forced backwards up into the prostate at the time of urination[8,9].

Magnetic infra red laser has analgesic effect due to changes in the potential of nerve cell membranes lead to its hyperpolarization as a good analgesic mechanism. It improves release reaction of endorphins and changes in transmitter concentration at the synapses $[9,10,11]$.

MIL also has inflammatory effect due to proliferation of immune cells (enhancement of immune response, increase of NK cell activity etc.), enhances lymphatic activity (drainage). It improves microcirculation (vasodilation) and reduces swelling (oedema \& haematoma resorption) $[9,10,11]$.

\section{Materials and methods:}

This study was an observational study done on 30 male patients with pelvic pain and age range varied from 30 to 60 years. The study period was from 2009 to 2011 in a private medical center at Dhaka city. The MIL-therapy in use has a wave length of $904 \mathrm{~nm}$ and a frequency of $3000 \mathrm{~Hz}$. The Laser beam reaches the prostate with a special optic probe. In this study the patient is examined per rectally after doing digital rectal examination with use of gloves and gel. It can be sterilized and it is atoxic. At the beginning we used a "Laser Super Sonic" machine with endorectal probe according to Strada. The treatment schedule was one treatment every two days (treatment's time of $5 / 10$ minutes, wave length $1000 \mathrm{KHz}$ )) for a total of 10 applications. Transrectal laser therapy was not indicated in prostate larger than $4 \mathrm{~cm}$ because this is the maximum depth of the laser beam's efficacy.

\section{Results:}

Out of 30 cases studied, age of the patients was between 30 to 60 years. Among the symptoms of the patients, dysuria was observed after applying MIL therapy.

Table-I shows age distribution of the patients.

Table- I

Distribution of patients by age $(n=30)$

\begin{tabular}{lcc}
\hline $\begin{array}{l}\text { Age of the } \\
\text { patients }(\mathrm{yrs})\end{array}$ & $\begin{array}{c}\text { Number of } \\
\text { patients }(\mathrm{n})\end{array}$ & $\begin{array}{r}\text { Percentage } \\
(\%)\end{array}$ \\
\hline $31-40$ & 13 & 43 \\
$41-50$ & 8 & 27 \\
$51-60$ & 9 & 30 \\
\hline
\end{tabular}


Table-II

Distribution of patients by symptoms $(n=30)$

\begin{tabular}{lcc}
\hline Symptoms & Number of patients & Percentage $\%$ \\
\hline Perineal Heaviness & 22 & 73 \\
Frequency & 22 & 73 \\
\hline
\end{tabular}

Among 30 patients 22 patients had Perineal Heaviness and 22 had frquency.

Table-III:

Digital rectal examination (DRE) finding of the study $\operatorname{cases}(n=30)$

\begin{tabular}{lcc}
\hline DRE finding & Number of patients & Percentage \\
\hline Enlarged prostate & 7 & 23 \\
Normal sized prostate & 23 & 77 \\
Any other finding & 0 & 0 \\
\hline
\end{tabular}

After DRE, among 30, 23 patients found normal sized prostate and only 07 patients found enlarged prostate.

Table-IV

Ultrasonogram (USG) finding of the patients(n)

\begin{tabular}{lcc}
\hline USG finding & Number of patients & Percentage \\
\hline Increased PVR & 7 & 23 \\
Enlarged prostate & 9 & 30 \\
Normal sized prostate & 21 & 70 \\
Any other & 0 & 0 \\
\hline
\end{tabular}

Table IV showing USG findings and found 21 patients had normal sized prostate and only 09 patients had enlarged prostate .07 patients found increased PVR

Table-V

Improvement of Perineal Heaviness and frequency after laser treatment

\begin{tabular}{lccc}
\hline Symptoms & Cured & Improved & Not improved \\
\hline Perineal Heaviness & 20 & 02 & 0 \\
Frequency & 22 & 0 & 0 \\
\hline
\end{tabular}

After per rectal laser therapy by optical probe there were improvement of symptoms found. Patients with Perineal Heaviness, about 20 were cured, 02 were improved and Patients with Frequency all (22) were cured.
Table-Vl

Complications after laser treatment $(n=30)$

\begin{tabular}{lcc}
\hline Significant complication & $\begin{array}{c}\text { Number of } \\
\text { patients }\end{array}$ & Percentage \\
\hline Yes & 0 & 0 \\
No & 30 & 0 \\
\hline
\end{tabular}

There were no significant complication found after per rectal laser treatment.

\section{Discussion:}

Chronic pelvic pain syndrome (CPPS), is used to designate unexplained chronic pelvic pain in men. This pain is associated with irritative voiding symptoms and/ or heaviness in the perineum, groin etc. Current research has provided evidence of numerous symptoms considerations, including frequency and perineal heaviness. Chronic prostatitis/Chronic pelvic pain syndrome (CPPS) is a very uncomfortable condition affecting males from very young age to above 70 s. The exact clean cut diagnosis is very difficult. Many patients of such condition end up with unnecessary protatectomies and bladder neck incisions(BNI), having no effective recovery. This made the scientists and urologists desire to find out ways of recovery from this benign but devastating situation.

Magnetic Infra Red Laser therapy(MIL-therapy) is a possible answer to alleviate this problem. This technique has not gained much popularity in our country as yet. Awareness of its effect having minor or no complication and further study are needed to gain its acceptance by clinicians.

Many investigators studied considering the symptomatology, perineal heaviness and frequency are the most common presentation which were present in $100 \%$ of the case studied. This correlates exactly with other different studies[9,10,11]. Regarding frequency and perineal heaviness are exactly similar to certain other studies[12,13,14].

Several studies showed that the symptoms perineal heaviness and frequency commonly cured by MIL therapy and some patients improved their symptoms after MIL therapy[11,12,5,16]. Digital rectal examination (DRE) is necessary mainly to exclude LUTS due to prostatic enlargement- benign or malignant ${ }^{14}$. Prostate size was normal in $77 \%$ cases and mild enlargement in $23 \%$ cases.

Post void residual volume(PVR) and prostate size were found to be normal in most of the cases in this series. Literature also illustrates the same findings[10,11]. 
Improvement of Symptoms of Chronic Prostatitis after MIL therapy

After completion of laser therapy, patients were assessed for their improvement and they were followed up for a period of six months. Improvement of symptoms were graded on a subjective scale of cured, improved and not improved. As the scoring is subjective, there is an element of fallacy. It was found that, there was hardly any patient who did not improve. Majority were cured and rest had improvement with only one patient having no improvement of any symptom. Studies in Europe and America describes around $100 \%$ recovery after laser therapy[14,15,16]. Similar studies showed totally cured the symtomps of Chronic prostatis after laser theray in Banhladesh[17,18].

\section{Conclusion:}

Magnetic infra red laser therapy is a very common and beneficial treatment of the patients of Chronic pelvic pain syndrome (CPPS). Laser Therapy is a safe and effective form of therapy.

This article produce a very good summary of the fruitful effect of laser therapy. The condition is poorly understood and difficult to treat.Among many sign and symptoms of CPPS, Perineal heaviness and frequency were observed and found very good effect after laser therapy. In this study it was proved that improvement and cure of perineal heaviness and frequency occur after applying MIL therapy .

\section{Conflict of Interest : None Declared}

\section{References:}

1. (http://www.finetreatment.co.uk/natural-alternative/ prostate-treatment.html)

2. Nickel JC. Pathogenesis of Chronic Protatitis. $\mathrm{Br}$ J Urol; 1990; 66: 47-49.

3. R.B. Alexander, K.J. Propert, A.J. Schaeffer, J.R. Landis, J.C. Nickel, M.P. O'Leary et al; Treating Men with Chronic Prostatitis/Chronic Pelvic Pain Syndrome, Annals of Internal Medicine; 2004, 141: 581-589.

4. Litwin MS. The National Institute of Health Chronic Prostatitis Symptom Index: development and validation of a new outcome measure. Chronic Prostatitis Collaborative Research Network. J Urol 1999; 162: 369-375.

5. Nickel JC, Nyberg LM, Hennenfent M. Research guidelines for chronic prostatitis: consensus report from the first National Institutes of Health
International Prostatitis Collaborative Network. Urology 1999; 54: 229-233. .

6. Ammerican top urologists Wikipedia

7 Top of Form. Mené MP, Ginsberg PC, Finkelstein LH, Manfrey SJ, Belkoff L, Ogbolu F. Transurethral microwave hyperthermia in the treatment of chronic nonbacterial prostatitis. J Am Osteopath Assoc. 1997; 97(1):25-30.

8. file:///C:/DocumentsandSettings/user/Desktop/ CPPS/NationalGuideline/Chronic pelvic pain_htm

9. Meares EJ. Prostatitis. Med Clin North Am 1991; 75:405-407.

10. Berghuis JP. Psychological and Physiological factors involved in chronic idiopathic prostatitis. J Urol 1998; 159: 1422- 1433.

11. McNaughton W. Chronic prostatitis: a thorough search for etiologically involved microorganisms. Infection 1999;19:119-125.

12. Krieger WW, Jacob J, Ross SO. Detecting urethral and prostatic inflammation in patients with chronic prostatitid. Urology 2000; 55: 186-192.

13. Barbalias GA. Clinical and therapeutic guidelines for chronic prostatitis. Letter to the Editoe. Eur Urol 2000; 37: 116-117.

14. Dimitrakov J. Recent developments in diagnosis and therapy of the prostatitis syndromes. Curr Opinion Urol 2001; 11: 87-91.

15. Moon TD. Questionnare survey of urologists and primary care physicians' diagnostic and treatment practices for prostatitis. Urology 1997; 50: 543-544.

16. Cho IR. Prostatic blood flow characteristics in the chronic prostatitis/ chronic pelvic pain syndrome. J Urol 2000; 163: 1130-1133.

17. Choudhury AMAR, Parveen T, Islam MF,Khan SA.A Recent Treatment of Chronic Prostatisand Laser Therapy.J Uttara Adhunick Med Coll.2013;3(1): 71-74.

18. Choudhury AMAR,Islam MW,Parveen T,Sultana P.Bangladesh J.Urol.2014;17(1):42-46.

\section{Abbreviations: \\ CPPS : Chronic Pelvic Pain Syndrome \\ EPS : Expressed Prostatic Secretion \\ ML : Magnetic Infrared Laser}

